\title{
Non-additive QTL mapping of lactation traits in 124,000 sequence- imputed cattle reveals novel recessive loci
}

Edwardo GM Reynolds¹, Thomas Lopdell², Yu Wang², Kathryn M Tiplady ${ }^{1,2}$, Chad S Harland², Thomas JJ Johnson², Catherine Neeley², Katie Carnie², Richard G Sherlock², Christine

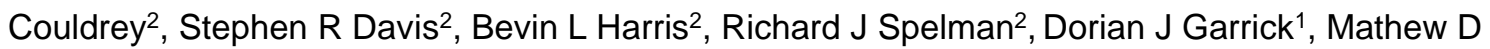
Littlejohn $n^{1,2}$

1. Massey University, New Zealand

2. Livestock Improvement Corporation, Hamilton, New Zealand

Correspondence should be addressed to EGMR (edwardo.reynolds.1@uni.massey.ac.nz) 


\section{Abstract}

2 Deleterious recessive conditions have primarily been studied in a Mendelian disease context.

3 Recently, several large effect, deleterious recessive mutations were discovered via non-additive

4 GWAS of quantitative growth and developmental traits in cattle. This showed quantitative traits

5 can be used as proxies of genetic disorders if they are indicative of whole animal health status

6 and susceptible to underlying genetic conditions. Lactation traits might also reflect genetic

7 disorders in cattle, given the increased energy demands of lactation and the substantial stresses

8 imposed on the animal. Here, we report a screen of over 124,000 cows for recessive effects

9 based on lactation traits. We discovered novel loci associated with five large recessive impacts

10 on milk yield traits represented by missense variants (DOCK8, IL4R, KIAA0556, and SLC25A4)

11 or premature stop variants (ITGAL, LRCH4, and RBM34) as candidate causal mutations. On milk

12 composition traits, we identified several small effect dominance contributions to previously

13 reported additive QTL. In contrasting analyses of milk yield and milk composition phenotypes, we

14 note differing genetic architectures. Milk yield phenotypes presented lower heritabilities and fewer

15 additive QTL, but higher non-additive genetic variance and a higher proportion of loci exhibiting

16 dominance compared to milk composition phenotypes. Large-effect recessive QTL are

17 segregating at surprisingly high frequencies in cattle. We speculate that the differences in genetic

18 architecture between milk yield and milk composition phenotypes derive from underlying

19 dissimilarities in the cellular and molecular representation of these traits. Lactation yields may act

20 as a better proxy than milk composition traits for a wide range of underlying biological disorders

21 affecting animal fitness

\section{Background}

25 Non-additive genetic effects are best known and studied in Mendelian disease contexts, where

26 recessive conditions have been shown to have major deleterious impacts on the health and

27 performance of animals. These studies have mostly used a 'forward genetics' approach, where

28 observation of a disease phenotype precedes fine mapping and sequencing to highlight the 
29 mutation [1-3]. The reverse approach has also been applied, where candidate loss of function

30 genotypes were identified and phenotyping was subsequently conducted to detect the impact of

31 the mutation [4,5]. Though examples remain limited, genome-wide association approaches have

32 been used to investigate non-additive effects in quantitative traits. Recent studies include the

33 investigation of complex traits in both humans [6] and cattle [7-11]. Reynolds et al. identified

34 several recessive mutations in cattle with major negative impacts on growth and developmental

35 traits, where some of these loci represented underlying genetic disorders [11].

37 The concept of using routinely gathered, quantitative traits as proxies of genetic disorders is 38 based on the idea that phenotypes such as growth or liveweight can be indicative of whole animal

39 health status, where reduced growth might be due to some underlying genetic disorder, and that

40 those effects could be detected via GWAS. It is therefore of interest to consider what other traits

41 might serve as proxies of animal fitness, with a view to extend the utility of this approach.

42 Lactation traits such as milk volume comprise one of the most commonly targeted classes of

43 quantitative traits studied in cattle, where additive analyses of these traits have presented

44 numerous candidate causative genes such as DGAT1 [12], GHR [13], ABCG2 [14], GPAT4 [15],

45 and MGST1 [16]. Lactation traits might also be reflective of genetic disorders, given the increased

46 energy demands of lactation and the substantial metabolic and physiological stresses imposed on

47 the animal [17]. We wondered therefore whether the application of non-additive models to

48 lactation data might identify further recessive mutations, and to this end, have conducted non-

49 additive GWAS for milk traits in 124,000 animals. We contrast the additive and non-additive

50 genetic architectures of milk yield traits and milk composition traits. Finally, we describe the

51 discovery of novel major effect recessive loci, highlighting candidate mutations that potentially

52 underlie undiagnosed recessive disorders.

\section{Methods}

\section{Animal populations}


57 The dataset reported in this study consists of 124,364 New Zealand dairy cattle. These animals

58 come from a mixed breed population, where 20,893 are 16/16 th's Holstein-Friesian (HF), 13,184

59 are $16 / 16^{\text {th's }}$ Jersey $(\mathrm{J}), 67,520$ are crosses involving varying proportions of the two breeds

60 (HFXJ), and 22,767 are HF or $\mathrm{J}$ crossbreeds with minor proportions of other breeds including

61 Ayrshire, Brown Swiss, or Hereford (and other crosses). An individual's breed may be coded as

$6216 / 16^{\text {th }} \mathrm{s}$, however, this does not preclude the possibility that an ancestor may be crossbred as

63 matings between $15 / 16^{\text {th }}$ s and $16 / 16^{\text {th }}$ s animals result in $16 / 16^{\text {th }}$ s offspring. The animals were

64 born between 1990 and 2018 with a mean birth year of 2010.

\section{Phenotypes}

67 Five first-lactation milk phenotypes were investigated in this study. These include three milk yield 68 traits; milk volume (L/Lactation; a lactation refers to a standardised 268 day lactation; $N=$

69 124,356), milk protein yield ( $\mathrm{kg} /$ Lactation; $\mathrm{N}=124,356)$, and milk fat yield ( $\mathrm{kg} /$ Lactation; $\mathrm{N}=$

$70124,356)$, and two milk composition traits; milk protein percentage $(\% ; N=124,363)$, and milk fat

71 percentage $(\% ; N=124,363)$. Milk protein yield and milk fat yield are the product of the milk

72 volume multiplied by the milk protein percentage or milk fat percentage, respectively.

73 Prior to genetic analysis, phenotypes were adjusted based on effects obtained from the national

74 genetic evaluation of the entire cattle population (30 million animals) which fits mixed linear

75 models. Fixed effects in that model included contemporary group, age at calving, stage of

76 lactation, and record type (records may be made at am milkings, pm milkings, or both). Since

77 animals have varying numbers of herd-test measurements within each milk trait, these were

78 aggregated to a phenotypic deviation such that each animal has a single record and a

79 corresponding weighting reflecting the amount of information in the record [18].

81 Sequence-based imputation reference panel

82 Whole genome sequencing was performed on 1,300 animals that were mostly ancestral sires,

83 these animals comprised the reference population for sequence-based imputation. Animals

84 comprising HF ( $\mathrm{N}=306), \mathrm{J}(\mathrm{N}=219), \mathrm{HFXJ}(\mathrm{N}=717)$, or other breeds and crossbreeds $(\mathrm{N}=58)$ 
85 were sequenced on Illumina HiSeq 2000 instruments targeting 100bp paired-end reads.

86 Sequence data were aligned to the ARS-UCD1.2 reference genome assembly using BWA 0.7.17

87 [19] resulting in a mean read depth of 15x. Variant calling was performed using GATK v4.0.6.0

88 [20], followed by variant filtering via Variant Quality Score Recalibration. Using animals with high

89 read depth (>10x, $N=850)$, variants were filtered out if they were singletons, were multi-allelic,

90 had a map quality score lower than 50 , or had a Mendelian error rate above $5 \%$. These criteria

91 left 21,005,869 whole genome sequence variants from the 850 highest read depth animals,

92 where these positions were then extracted from the sequence data on all 1,300 animals and

93 phased using Beagle 5.0 [21] to create the sequence-based imputation reference panel.

95 Genotyping

96 The study animals $(N=124,364)$ were genotyped using SNP chips, where either ear-punch

97 tissue samples or blood samples were used for DNA extraction. Genotyping was performed using

98 a variety of platforms including GeneSeek GGPv1, GGPv2, GGPv2.1, GGPv3, GGPv3.1, GGPv4,

99 GGP50kv1, GGP50kv1.1, Illumina BovineSNP50v1, Illumina BovineSNP50v2, or BovineHD SNP-

100 chips. Samples were processed for DNA extraction at GeneMark (Hamilton, New Zealand) using

101 Qiagen BioSprint kits or GeneSeek (Lincoln, NE, USA) using Life Technologies' MagMAX

102 system.

103

104 Consolidation of SNP-chip panels for sequence imputation

105 Imputation from genotyping panels to sequence resolution was performed as described in Wang

106 et al. [22]. Genotype panels were grouped into four sets; GGP panels (GGPv1, GGPv2,

107 GGPv2.1, GGPv3, GGPv3.1, and GGPv4), 50K panels (BovineSNP50v1, and BovineSNP50v2),

108 GGP50k panels (GGP50kv1, GGP50kv1.1), and the BovineHD panel. Animals genotyped on the

109 GGP panels were imputed to the BovineSNP50v1 panel, then combined with the physically

110 genotyped 50K panel animals and further imputed to the BovineHD panel. Animals genotyped on

111 the GGP50k panels were separately imputed to the BovineHD panel. In order to incorporate the

112 large amount of custom content genotyped on the GGPv3 platform, we conducted similar 
113 imputation steps to impute all animals to GGPv3. We then combined the imputed and physically

114 genotyped panels (imputed HD, imputed GGPv3, and physically genotyped HD), and imputed

115 these animals to sequence resolution using the sequence-based imputation reference population,

116 described above. Post-imputation filtering to remove very rare variants (homozygous alternate

117 count $\leq 5$ ) was performed, as well as a filter to remove variants that imputed poorly based on the

118 dosage $\mathrm{R}^{2}$ statistic $\left(\mathrm{DR}^{2} ; \mathrm{DR}^{2}<0.7\right)$. After the application of these filters, 16,640,294 variants

119 remained for GWAS and further analysis.

120

121 Genotypes for population structure adjustment

122 We used content from the Bovine SNP50 chip platform to account for the population structure of

123 the sample. From the initial 54,708 autosomal SNPs, we filtered to remove markers with high

124 missing genotype rates $(>0.01)$, low minor allele frequency $(<0.02)$, or high deviations from

125 expected Hardy-Weinberg equilibrium ( $>0.15$, calculated within breed). This was followed by

126 further filtering to remove markers that appeared to impute poorly $\left(D R^{2}>0.9\right)$, and markers in

127 high LD with another marker on the panel (pairwise $R^{2}>0.9$, within $1 \mathrm{Mbp}$ ). These criteria

128 resulted in a set of 31,451 SNP chip markers for subsequent analysis.

\section{Heritability estimates}

131 We estimated breed-specific additive and dominance heritabilities using genomic relationship

132 matrices (GRMs) using GCTA software $[6,23]$. Variance components were estimated from

133 purebred individuals ( $\mathrm{HF}=20,893, \mathrm{~J}=13,184$ ), using the same set of 31,451 filtered

134 BovineSNP50 SNPs used for population structure adjustment (filters described in the previous

135 section). GCTA estimates variance components using a restricted maximum likelihood (REML)

136 approach, where additive heritability $\left(h^{2}\right)$ is the ratio of additive genetic variance to phenotypic

137 variance, and dominance heritability $\left(\delta^{2}\right)$ is calculated as the ratio of dominance genetic variance

138 to phenotypic variance. 


\section{Model Overview}

142 We applied a non-additive GWAS method similar to that described in Reynolds et al. [11] to

143 identify non-additive QTL for milk traits. This two-step method first uses a leave-one-segment-out

144 (LOSO) approach to fit genomic marker effects to adjust for population structure, and a second-

145 step Markov chain Monte Carlo (MCMC) method to test the effects of all imputed-to-sequence

146 variants, one at a time. In general, for each sequence variant the method fits the following model:

$$
y=1 \mu+T b+M_{\alpha} \alpha+M_{\delta} \delta+e
$$

148 Where $\boldsymbol{y}$ indicates a vector of one of the 5 phenotypes of interest, pre-adjusted as described in

149 the 'Phenotypes' section above, $\boldsymbol{\mu}$ is the overall mean, $\boldsymbol{1}$ is a vector of ones, $\boldsymbol{b}$ is a vector of

150 genotype class effects for the sequence variant of interest, and $\boldsymbol{T}$ is the design matrix relating

151 records to genotype class for the sequence variant. The vector $\boldsymbol{\alpha}$ represents random SNP chip

152 additive marker effects spanning the whole genome except the segment of interest such that $\boldsymbol{\alpha} \sim$

$153 \mathrm{~N}\left(\boldsymbol{O}, \boldsymbol{I}_{\alpha^{2}}\right)$, where $\boldsymbol{I}$ is an identity matrix of order equal to the number of marker effects and $\sigma_{\alpha^{2}}$

154 represents the additive marker effect variance, $\delta$ is a vector of random SNP chip dominance

155 marker effects spanning the whole genome except the segment of interest such that $\delta \sim \mathrm{N}(\boldsymbol{0}$,

$\left.156 \boldsymbol{I}_{\delta}{ }^{2}\right)$, where $\sigma_{\delta}^{2}$ represents the dominance marker effect variance. $\boldsymbol{M}_{\boldsymbol{\alpha}}$ and $\boldsymbol{M}_{\boldsymbol{\delta}}$ are matrices with

157 each column representing the covariate values for a marker locus $([0,1,2]$ and $[0,1,0]$,

158 respectively). The vector $\boldsymbol{e}$ represents residuals with $\boldsymbol{e} \sim \mathrm{N}(\boldsymbol{O}, \boldsymbol{R})$, where for a simple model based

159 on single observations $\boldsymbol{R}=\boldsymbol{I} \sigma_{\mathrm{e}}^{2}$, where $\boldsymbol{I}$ is an identity matrix of order equal to the number of

160 phenotypic records and $\sigma_{\mathrm{e}}^{2}$ represents the residual error variance. Since the traits investigated

161 here are represented by the mean of a variable number of repeated observations, the diagonal

162 elements of $\boldsymbol{R}$ varied according to the number of observations contributing to the yield deviation.

163 One notable contrast to the model implemented in Reynolds et al., is that in the current model, we

164 fit both additive $\left(\mathrm{M}_{\alpha}\right)$ and dominance $\left(\mathrm{M}_{\delta}\right)$ effects of the genomic markers to adjust for population

165 structure. This modification was made to better control the inflation observed when analysing milk

166 traits in a population larger than that studied in Reynolds et al. [11]. 
168 Population structure adjustment

169500 samples of vectors of plausible marker effects, $\widetilde{\boldsymbol{\alpha}}$ and $\widetilde{\boldsymbol{\delta}}$, for the 31,451 SNP-chip markers,

170 were generated using single-site Gibbs sampling from an extension of the BayesC 0 algorithm

171 implemented in GenSel using standard priors [24]. That algorithm was performed while omitting

172 the $\mathbf{T b}$ term from (1) and convergence of the Markov chain of plausible marker effects was

173 determined using the Geweke diagnostic [25]. LOSO was used to avoid fitting SNP-chip marker

174 effects in linkage disequilibrium with the sequence variant being tested. The genome was

175 partitioned into 10Mbp LOSO intervals and, for each interval, phenotypes were adjusted for the

176 samples of SNP chip marker effects except those within the relevant LOSO interval. This

177 produced distinct LOSO-adjusted phenotypic deviations for each 10Mbp interval for each sample

178 of plausible marker effects.

179

180 Association analysis

181 For each sequence variant, we sampled genotype class effects for each plausible sample of

182 LOSO-adjusted phenotypic deviations. We obtained MCMC chains of additive and dominance

183 genotypic effects, and standard-additive effects as contrasts of these plausible genotype class

184 effects. These posterior distributions were summarised by their posterior means, posterior

185 standard deviations, and z-statistics following a standard Normal distribution [26]. The statistical

186 significance of standard-additive, additive, and dominance genetic effects were evaluated using a

187 Z-test.

189 QTL identification, significance criteria, and annotation

190 We primarily aimed to detect non-additive QTL, as such we declared variants significant if the

191 dominance genotypic effect, $\mathbf{d}$, passed a false discovery rate (FDR) threshold of $1 \times 10^{-3}$. For each

192 phenotype, this FDR threshold was calculated using q-values [27] as implemented in the qvalue

193 package in R [28]. Since we were particularly interested in medium- to large-effect QTL, only loci

194 with effect sizes (a, or d) greater than 5\% the phenotypic standard deviation of the trait were 
195 considered for further downstream analyses. We calculated the dominance coefficient $k=\frac{d}{|a|}$ for

196 each significant QTL to characterise the non-additive mechanism presented, where $k \approx 0$

197 represents a completely additive locus, $k \approx 1$ represents a completely recessive locus, $k<1$ a

198 partially dominant locus, and $k>1$ an over-dominant locus.

199 For standard-additive effects, $\boldsymbol{\alpha}$, we used GCTA-COJO [29] to detect tag variants for QTL

200 identified in our standard-additive GWAS. GCTA-COJO utilises LD structure and GWAS

201 summary statistics to iteratively identify significant QTL at the FDR threshold of $1 \times 10^{-3}$. We used

202 sequence annotations from variant effect predictor (Ensembl 97, [30]) to highlight mutations that

203 might be responsible for non-additive QTL identified, where the potential impact of missense

204 mutations on protein function was judged using SIFT scores [31].

\section{Iterative GWAS}

207 We aimed to investigate whether multiple dominance QTL might segregate at associated loci, so

208 implemented an iterative GWAS approach to differentiate QTL. Here, we first identified the

209 variants on each chromosome that surpassed the false discovery threshold. We then adjusted the

210 phenotype for the genotype class effects of the most significant variant (or candidate causal

211 variant if identified) and then re-ran the GWAS model on the chromosome of interest using the

212 residual phenotype. This process was iterated until there were no further significant QTL on the

213 chromosome.

\section{Results}

\section{Heritabilities of lactation traits}

218 We first estimated additive and dominance heritabilities for each phenotype within each breed to

219 investigate the additive and non-additive genetic architecture of each trait. These results are

220 shown in Table 1, additive heritabilities far outweighed dominance heritabilities, though presented

221 ratios of similar magnitude to those previously reported for other traits and populations [8,32]. Milk 
222 fat yield in Jersey cows had the highest dominance heritability at 0.074 , and milk protein

223 percentage in Holstein-Friesian cows had the lowest dominance heritability at 0 . Of note, a

224 distinct contrast in relative heritabilities was apparent between milk composition and milk yield

225 traits, where composition traits had high additive heritabilities but near zero dominance

226 heritabilities, and yield traits presented lower additive heritabilities but higher dominance

227 heritabilities (Table 1).

228

\begin{tabular}{|c|cc|cc|}
\hline Table 1 | Heritability estimates for lactation traits \\
\hline Trait & \multicolumn{1}{l|}{$\mathrm{h}^{2} \mathrm{HF}^{2}$} & $\delta^{2} \mathrm{HF}_{1}$ & \multicolumn{1}{l|}{$\mathrm{h}^{2}{ }_{\mathrm{J}}$} & $\delta^{2} \mathrm{~J}$ \\
Milk Volume & 0.296 & 0.044 & 0.312 & 0.064 \\
Milk-Fat Yield & 0.261 & 0.059 & 0.232 & 0.074 \\
Milk-Protein Yield & 0.235 & 0.053 & 0.236 & 0.073 \\
Milk-Fat Percentage & 0.7 & 0.006 & 0.616 & 0.015 \\
Milk-Protein Percentage & 0.642 & 0 & 0.636 & 0.005 \\
\hline
\end{tabular}

$\mathrm{h}^{2}$ - Additive heritability, $\delta^{2}$ - dominance heritability, HF - Holstein-Friesian, J Jersey

$230 \quad$ Lactation trait GWAS

231 We performed GWAS' across the five milk traits of interest, namely milk volume, milk protein

232 yield, milk fat yield, milk protein percentage, and milk fat percentage to identify non-additive QTL

233 (Figure 1). Both additive and dominance effects are included in these plots, where iterative

234 analysis identified 23 dominance QTL signals that passed our FDR threshold. These included 10,

$23511,12,8$, and 7 QTL from 4,618, 2,706, 8,525, 8,987, and 5,800 significant variants across milk

236 volume, milk protein yield, milk fat yield, milk protein percentage, and milk fat percentage,

237 respectively. These signals spanned 13 discrete autosomes. In standard-additive GWAS,

238 following iterative COJO analysis, we identified 217, 152, 142, 673, and 457 QTL across milk

239 volume, milk protein yield, milk fat yield, milk protein percentage, and milk fat percentage,

240 respectively.

241

242 Dominance QTL 
243 We identified 15 significant dominance QTL for milk yield traits, and 11 for milk composition traits

244 (Table 2, Supplementary Table 1). Across the milk yield dominance QTL, the majority $(\mathrm{N}=12)$

245 were recessive effects and they were located on chromosomes 2, 4, 5, 8, 12, 25, 28, and 29.

246 Seven of these signals appear to be novel to the current study, the remainder having been

247 recently highlighted in our analysis [11] of growth and developmental traits in an overlapping

248 population to that described here. Across the 11 milk composition dominance QTL, the majority

$249(\mathrm{~N}=8)$ presented partial dominance effects, with six of these representing loci identified from

250 previously published additive GWAS (Supplementary Table 1).

251 Figure 2a contrasts the minor allele frequency and effect size of dominance components for all of

252 these effects. Interestingly, milk composition trait QTL appeared to be tagged by high minor allele

253 frequency variants with comparatively small effect sizes, whereas milk yield QTL tag variants had

254 low minor allele frequencies and larger effects. The type of effects also appeared to differ

255 between traits (Figure $2 \mathrm{~b}$ ), where we noted an abundance of recessive QTL in milk yield traits,

256 whereas milk composition traits mostly comprised partially dominant QTL. 
bioRxiv preprint doi: https://doi.org/10.1101/2021.08.30.457863; this version posted September 1,2021 . The copyright holder for this preprint (which was not certified by peer review) is the author/funder. All rights reserved. No reuse allowed without permission.

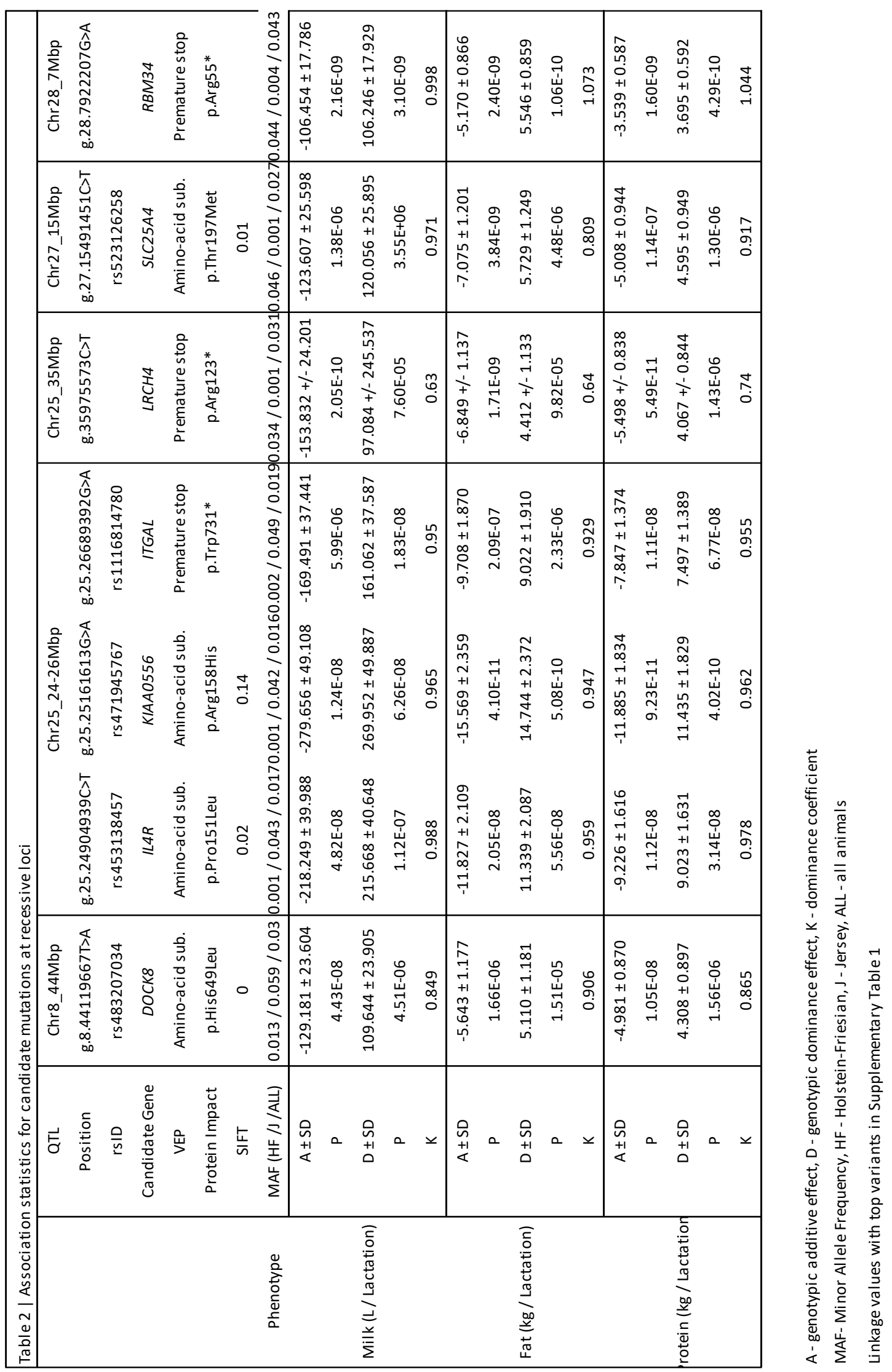




\section{Candidate causal mutation identification}

259 Given the status of recessive milk yield QTL as potentially representing novel bovine disorders,

260 we prioritised these QTL for further investigation, selecting QTL where the dominance coefficient

261 (k) was near $1(0.7<k<1.3)$. We used sequence annotations from variant effect predictor to

262 highlight mutations that might be responsible for these effects (Ensembl 97, [30]), highlighting

263 variants that were in strong to moderate $\operatorname{LD}\left(R^{2}>0.7\right)$ with the lead variant per locus, and that

264 were also predicted to alter or disrupt protein function. We identified 5 novel recessive QTL

265 (including one biologically compelling near-significant recessive QTL), and several other

266 recessive QTL previously identified and attributed to mutations in the PLCD4, FGD4, MTRF1,

267 GALNT2, DPF2, and MUS81 genes [11]. Figure 3 presents the position, regional LD, and

268 association statistics for the QTL novel to the current study. Note that we have applied relatively

269 simple annotation criteria and only highlight protein-coding variants as candidates since, for

270 recessive signals at least, we consider protein altering mutations primary candidates given the

271 loss of function connotation for these effects. Supplementary Table 1 shows all significant QTL

272 identified, including those not expanded upon here.

\section{Chromosome 8}

275 Chromosome 8 presented a significant signal at 45Mbp for milk protein yield and milk fat yield.

276 The most significant variants for these signals ( $\mathrm{g} .45878531 \mathrm{~A}>\mathrm{C}$ and $\mathrm{g} .45880948 \mathrm{C}>\mathrm{T}$ ) were in

277 strong LD $\left(R^{2}=0.99\right)$, and we note an annotated missense variant (g.44119667T $>A$,

278 rs483207034) in high LD with both top-associated variants $\left(R^{2}=0.85\right.$ and 0.85 , respectively;

279 Figure 3a). This variant in the DOCK8 gene results in an amino acid (p.His649Leu) change and

280 has a predicted deleterious impact $(\mathrm{SIFT}=0)$.

282 Chromosome 25

283 A dispersed QTL signal is apparent on chromosome 25 at 24-27Mbp across the three lactation

284 yield traits, with the top variants at g.25921991AT>T for milk fat yield, and g.27868969C >T for

285 milk protein yield and milk volume. Effect prediction highlighted three candidate causal mutations 
286 in the region. These included a p.Pro151Leu substitution in the IL4R gene (g.24904939C>T,

287 rs453138457) with $R^{2}=0.74$, and 0.62 , for the milk fat and milk protein/milk volume top variants,

288 respectively, another missense variant (p.Arg158His) in the KIAA0556 gene (g.25161613G>A,

289 rs471945767) with $R^{2}=0.89$, and 0.74 , respectively, and a nonsense variant ( $p$. Trp731*) in the

290 ITGAL gene (g.26689392G>A, rs1116814780) with $\mathrm{R}^{2}=0.76$, and 0.70, respectively (Figure 3b).

291 While these are plausible candidates to explain the QTL, we were not able to distinguish between

292 the candidates through iterative analysis, where fitting any one of these candidates removed the

293 majority of the association at this locus.

295 A second signal for protein yield on chromosome 25 was observed at $35 \mathrm{Mbp}$. This locus

296 maintained its significance after accounting for the chromosome $2525 \mathrm{Mbp}$ QTL through iterative

297 analysis, suggesting it was a discrete effect. The locus presented a strong candidate causative

298 mutation as potentially underlying the effect, comprising a stop gain mutation (g.35975573C>T;

$\left.299 \operatorname{Arg} 123^{*}\right)$ in the $L R C H 4$ gene that was the third most highly associated variant at this locus overall

300 (Figure $3 c)$. We observed a mostly recessive effect for this variant $(\mathrm{k}=0.74)$, where animals

301 carrying the heterozygote and homozygous alternate genotypes produce $1.44 \mathrm{~kg}$, and $11.21 \mathrm{~kg}$

302 less milk protein per lactation compared to the homozygous reference genotype. When fitting

303 g.35975573C>T as a fixed effect, the significance of the QTL is removed, and no further QTL are

304 apparent on the chromosome (Supplementary Figure 1).

306 Chromosome 27

307 We observed a signal at $15 \mathrm{Mbp}$ on chromosome 27 for milk protein yield. Although this did not

308 surpass our q-value FDR threshold of $1 \times 10-3$ (equivalent to $P=1.65 \times 10^{-7}$ ), this signal was

309 conspicuous given that the lead variant $\left(\mathrm{g} .15491451 \mathrm{C}>\mathrm{T}\right.$; rs523126258, $\mathrm{p}$-value $\left.=1.30 \times 10^{-6}\right)$ is a

310 predicted deleterious missense mutation ( $\mathrm{p}$.Thr197Met) in the SLC25A4 gene. Figure 3d shows a

311 Manhattan plot for this region.

312

\section{Chromosome 28}


314 We previously reported a major recessive bodyweight QTL on Chromosome 28 represented by a

315 likely causative splice acceptor mutation in GALNT2 (g.2281801G>A) [11]. This QTL was

316 apparent in the current analysis, impacting all three milk yield traits. However, the application of

317 iterative association analysis revealed a secondary QTL approximately 4Mb downstream of the

318 GALNT2 mutation at Chr28:6-7Mbp (top variant at g.6223350G>A). This residual signal

319 highlighted a stop-gain non-sense mutation (g.7922207G>A) strongly linked to the

320 g.6223350G $>$ A variant $\left(R^{2}=0.89\right.$; Figure 3e). This stop-gain mutation (p.Arg55*) is in the RBM34

321 gene, and appears to be in linkage equilibrium with the GALNT2 causal mutation $\left(R^{2}<0.001\right)$,

322 having little association with bodyweight in our previous analysis $(p=0.37 ;[11])$. Upon the second

323 chromosome 28 GWAS iteration (fitting both GALNT2 and RBM34 mutations as fixed effects),

324 there were no further significant QTL on the chromosome (Supplementary Figure 2).

326 Dominance QTL for composition traits

327 In addition to the recessive QTL identified for milk yield traits, we also identified dominance QTL

328 for milk composition traits. We investigated these effects and observed several partial dominance

329 QTL in close proximity to previously described additive loci. The tag variants of these QTL were

330 adjacent the genes; CSF2RB [33], MGST1 [16], DGAT1 [12], GHR [13], GPAT4 [15], and

331 PICALM [34] and, in each case, these variants were in high linkage disequilibrium $\left(R^{2}>0.8\right)$ with

332 previously identified causal and/or tag variants (Supplementary Table 1).

334 Milk protein percentage presented multiple dominance QTL on Chromosome 6 within the

335 Chr6:80-85Mbp region (Supplementary Table 1). The most significant of these QTL presented the

336 top variant g.84112451C>A and shows a partial dominance effect. Unlike the examples

337 highlighted above, no very strongly linked candidate mutation was identified, though we note that

338 this variant is in moderate LD with a previously proposed causative variant in $C S N 1 S 1\left(R^{2}=0.53\right.$;

339 p.Glu192Gly mutation; g.85427427A>G) [35]. Chromosome 12 presented a significant

340 dominance QTL, where we observed a partial dominance effect at $68 \mathrm{Mbp}$ for milk protein

341 percentage with the top variant at g.68763031T>TG. As with the chromosome 6 locus, no 
342 particularly obvious candidate causal variant or gene was identified that might account for this

343 signal.

345 Contrasting additive and dominance GWAS results

346 Figure 4 compares minor allele frequency (MAF) and the effect sizes between homozygous

347 genotypes across all traits and genetic mechanisms. As might be expected, we observed many

348 more additive QTL than dominance QTL across all traits. Notably however, mutations detected

349 via dominance GWAS in milk yield traits presented very large effects compared to the additive

350 QTL detected for these traits, and most presented a recessive mechanism. On the other hand,

351 the largest effects presented for the two milk composition traits were mostly additive QTL, where

352 dominance effects tended to be higher MAF and incompletely dominant in their presentation of

353 effect.

\section{Discussion}

357 The results in this study highlight the presence of many non-additive QTL for milk traits in cattle.

358 The majority of these signals for milk yield traits present recessive QTL, identifying five novel loci

359 and several previously described recessive QTL [11]. Although milk protein percentage and milk

360 fat percentage traits also yielded many dominance GWAS signals, most presented partially

361 dominant QTL that appeared to represent minor dominance components to previously reported

362 additive QTL.

364 Different trait classes present contrasting additive and non-additive genetic architectures

365 One remarkable observation from the current study is the apparent difference in additive and non-

366 additive genetic architectures between milk yield traits and milk composition traits. Dominance

367 heritabilities of yield traits ranged from $3 \%$ to $7 \%$, whereas composition traits have dominance

368 heritabilities at or near zero. By contrast, additive heritabilities ranged from $23 \%$ to $31 \%$ for yield 
369 traits, compared to composition traits which ranged from $64 \%$ to $70 \%$. These findings are

370 consistent with Sun et al. [8] where they observed similar additive and dominance heritabilities

371 and suggest dominance, in particular recessive mechanisms, may play a bigger role in the

372 regulation of yield traits than composition traits.

374 These architecture contrasts were also apparent when comparing the properties of individual

375 dominance QTL between milk yield and milk composition traits. Dominance QTL identified in milk

376 yield traits manifested primarily with recessive genetic mechanisms, while milk composition traits

377 presented primarily partial dominance effects. Of further note, milk yield trait dominance QTL

378 typically had low minor allele frequencies and large effect sizes, whereas dominance QTL for milk

379 composition traits were typically characterised by high minor allele frequencies and smaller effect

380 sizes. We theorise that these observations may be due to the way in which the different traits are 381 able to reflect underlying deleterious recessive syndromes - i.e., their utility to serve as proxies of

382 genetic disorders. Of all recessive QTL detected in the current study, we previously validated a

383 subset of these as representing new genetic disorders [11]. Although we did not investigate the

384 novel recessive loci in this study with the same rigour as those investigated in Reynolds et al.,

385 their very large, uniformly negative effects suggest some at least will similarly validate as new

386 recessive syndromes. Notably, none of these loci (new or old) show substantial effects on milk

387 composition, suggesting milk fat and protein percentage traits do not readily reflect recessive

388 effects. This finding can be rationalised by the comparatively broad range of biological processes

389 reflected by milk yield traits (or the growth and development traits investigated in Reynolds et al.

390 2021), where the energy demands of lactation (or growth) might be expected to manifest a wide

391 range of other organismal stresses. The relative composition of milk components, by contrast,

392 likely represents a narrower spectrum of mammary-specific biology that we hypothesise is less

393 able to serve as a proxy of animal fitness.

395 It should be acknowledged that given protein yield and fat yield are the products of milk volume

396 and their respective percentages, these traits are not independent. We observe the variance 
397 components and genetic architectures of milk fat yield and milk protein yield are more

398 comparable to milk volume than their respective composition traits. This suggests milk volume

399 has a greater influence on milk fat yield and milk protein yield due to the additional environmental

400 factors and measurement errors affecting milk volume.

401

402 Previous studies highlighting recessive effects on quantitative traits

403 As discussed above, we recently reported an investigation of growth and developmental traits 404 that identified non-additive QTL using similar approaches to those presented here [11]. That 405 study demonstrated how quantitative traits can be used as proxies to map genetic disorders 406 without prior disease identification. In doing so, using sequence-resolution variants, the research 407 highlighted several recessive QTL represented by variants in the PLCD4, FGD4, MTRF1, 408 GALNT2, DPF2, and MUS81 genes, each with large effects on bodyweight and other quantitative 409 traits. The work presented here builds on those findings; we identified many of the same 410 recessive mutations as well as several additional recessive QTL. The additional discoveries made 411 here can be assumed to reflect the increased sample size leveraged in the current study.

413 Aside from the Reynolds et al. study discussed above, few other studies have highlighted major 414 effect recessive impacts using quantitative trait data. Although non-additive GWAS with large 415 sample sizes has been performed in cattle [10,32], low marker densities in these earlier studies 416 may have hampered the ability to directly resolve candidate causative variants [11]. This 417 challenge arises due to the different linkage disequilibrium (LD) properties between causal and 418 observed variants for additive and non-additive QTL, where LD of an observed marker tagging a 419 causal variant will manifest at $\mathrm{R}^{2}$ for an additive effect, compared to $\mathrm{R}^{4}$ for a recessive signal.

420 This means observed tag variants need to be more closely linked to causal dominance variants to 421 capture the QTL [36,37]. Despite limited prior literature on the use of non-additive GWAS to this 422 end, one noteworthy study suggesting the importance of recessive variants to animal breeding 423 traits was recently reported in the context of male fertility and semen traits in cattle [38]. Here, the 424 researchers identified recessive QTL and candidate causal mutations in several genes including 
425 a missense variant in SPATA16. That study used imputed genotypes at high density (based on

426 the Illumina BovineHD platform), though it is noteworthy that the study population used was quite

427 small ( $N=3,736$ bulls). It seems likely that the discovery of these QTL was aided in part by the

428 remarkable frequency of the deleterious haplotypes identified in that study, presenting allele

429 frequencies ranging from 9-34\% [38].

430

\section{Recessive QTL of interest}

432 Although many non-additive signals were identified in this study, we were particularly interested in

433 recessive QTL with large effects, given that these might represent underlying genetic disorders.

434 The five novel recessive QTL on chromosomes 8, 25, 27, and 28 are presented and discussed

435 below.

436

437 Chromosome 8 - DOCK8

438 Our results present a missense mutation in the DOCK8 gene as potentially having a deleterious

439 recessive impact on milk yield traits. The QTL appears to operate in a completely recessive

440 manner, with the DOCK8 variant present at low allele frequencies in each breed (Holstein-

441 Friesian MAF $=0.013$, Jersey MAF $=0.059) . D O C K 8$, dedicator of cytokinesis 8 , is involved with

442 guanine nucleotide exchange factors and influences intracellular signalling networks, and is

443 important in immune responses and lymphocyte regulation in humans and mice [39]. Recessive

444 mutations in DOCK8 have been associated with hyper Immunoglobulin E syndrome leading to the

445 onset of combined immunodeficiency disease and other health complications [40]. In mice,

446 compromised immune responses are also observed including negative impacts on B cell

447 migration [41], and T cell migration and viability [42,43]. DOCK8 variants have not previously

448 been associated with cattle performance traits, though if this missense mutation underlies the

449 chromosome 8 QTL, it could be presumed to act through similar negative impacts on the immune

450 system. Under this hypothesis, it is unknown whether the lactation effects are due to mammary

451 immune function or secondary impacts, though given that higher levels of circulating

452 immunoglobulin E and lymphocyte profiling can indicate DOCK8 deficiency in humans $[40,44]$, 
453 future work to sample and profile homozygous animals could be used to definitively establish the

454 causality of the DOCK8 missense mutation for this QTL.

455

456 Chromosome 25 - IL4R, KIAA0556, ITGAL

457 The QTL identified on chromosome 25 at 24-27Mbp presented three candidate mutations in 458 genes: IL4R, KIAA0556, and ITGAL. IL4R, Interleukin 4 receptor, is a transmembrane protein

459 involved in immune responses in humans [45]. KIAA0556 is associated with microtubule

460 regulation in humans, and knockout mutations in humans and mice have been associated with

461 the neurological disorder, Joubert syndrome [46]. ITGAL encodes integrin alpha L chain, and loss

462 of function variants in this gene have been associated with compromised immunity including

463 increased susceptibility to infection to Salmonella in mice [47]. Given that iterative association

464 analysis failed to prioritise one of these variants over the other, it is unknown which of these

465 variants might be responsible for the QTL, and our focus on protein-coding variants as candidates

466 may have also overlooked alternative non-coding or structural mutations as responsible. These

467 variants are nevertheless in moderately strong, though not perfect $L D$ (max. pairwise $R^{2}=0.79$ ),

468 so physical genotyping for fine mapping and future functional testing should help to resolve the

469 identity of the gene (or genes) underpinning this QTL.

471 Chromosome $25-\mathrm{LRCH4}$

472 Although iterative GWAS did not resolve candidates in the above example, this approach did 473 highlight a second QTL on chromosome 25 represented by a nonsense mutation in the $L R C H 4$ 474 gene. $\mathrm{LRCH} 4$, leucine-rich repeats and calponin homology containing protein 4, regulates the 475 signalling of toll-like receptors (TLRs) and has been shown to influence innate immune responses

476 in mice [48]. In that study, researchers showed $L R C H 4$-silenced cells presented reduced

477 expression across pro-inflammatory cytokines produced in the TLR4 pathway, most notably that

478 of IL-10 and MCP-1. This suggests a knockout mutation, like that observed here, may have

479 negative impacts on innate immunity in cattle that may drive negative impacts on milk volume, 480 milk fat yield, and milk protein yield. 
482 Chromosome 27 - SLC25A4

483 While non-significant at the genome-wide level (c.f. $P=1.65 \times 10^{-7}$ vs $P=1.30 \times 10^{-6}$ ), the

484 chromosome 27 15.5Mbp locus presented a conserved amino acid mutation in SLC25A4 as the

485 lead associated variant and was therefore of note. This variant demonstrated a complete

486 recessive effect on all three lactation yield traits. The SLC25A4 gene, solute carrier family 25

487 member 4, encodes the Adenine nucleotide translocator (Ant1) protein, responsible for the

488 translocation of ATP and ADP between the cytoplasm and mitochondria. In mice, knockouts of

489 SLC25A4 result in mitochondrial myopathy and cardiomyopathy, and a severe intolerance to

490 exercise [49]. Similarly, in humans, childhood-onset mitochondrial disease and exercise

491 intolerance have been observed for both dominant [50] and recessive mutations [51] in SLC25A4.

492 If future association studies confirm the non-significant associations highlighted in the current

493 study, it would be intriguing to examine the phenotypes of homozygous cows further, given the

494 implication that mitochondrial functional deficits and exercise intolerance might underlie these

495 lactation performance impacts.

498 On first appearance, the strong associations with lactation yield traits near the beginning of

499 chromosome 28 might reasonably be attributed to the GALNT2 splice site mutation reported and

500 investigated previously [11]. However, upon fitting this mutation as a covariate in our iterative

501 GWAS approach, a secondary peak was still strongly apparent, highlighting a nonsense mutation

502 in the RBM34 gene as potentially responsible for the effect. The RBM34 gene encodes an RNA

503 recognition motif protein with an RNA-binding domain. There appears to be little previous

504 research in humans or model organisms on $R B M 34$, with limited recent literature probing its

505 involvement in embryonic stem cell differentiation [52]. Here we observed a predicted

506 homozygous knockout of the gene that may influence milk volume, milk protein yield, and milk fat

507 yield in a recessive manner, though its status as a largely uncharacterised RNA-binding protein

508 leaves little room for speculation as to how those effects might manifest. Mechanism aside, 
509 identification of two uncorrelated recessive QTL demonstrates the utility of using iterative GWAS

510 approaches, given that 'peaks' with compelling causative mutations presented by previous

511 analyses might otherwise go un-investigated. Of further note at this locus, other researchers

512 appear to have observed lactation effects at the $6-10 \mathrm{Mb}$ locus previously [53]. However, there

513 appears to be very low $L D\left(R^{2}\right.$ with $R B M 34=0.04$, GALNT2 $\left.=0.02\right)$ between the tag variant

514 identified by Raven et al. (rs41607517) and the nonsense mutations identified here, suggesting

515 these are likely different effects.

517 Previously described additive QTL present partial dominance

518 We observed several partial dominance QTL closely linked to previously described QTL identified

519 from standard-additive analyses. As described in Supplementary Table 1, we identified

520 dominance components in high LD with variants associated with the genes; CSF2RB [33],

521 MGST1 [16], DGAT1 [12], GHR [13], AGPAT6 [15], PLAG1 [54,55], and PICALM [34] (and in

522 moderate LD with a CSN1S1 variant [35]). These partial dominance associations were mostly

523 identified in percentage traits. These observations suggest that many well-known major-effect

524 QTL identified from additive analyses incorporate some level of non-additivity, in agreement with

525 the analyses of milk traits reported by Jiang et al. $(2017 ; 2019)[10,32]$.

\section{Conclusion}

528 In this study, we have highlighted that different classes of lactation traits (yield compared to

529 composition traits) present differing additive and non-additive genetic architectures. We

530 speculate, that these differences derive from underlying contrasts in the cellular and molecular

531 representation of these traits, where despite comparatively low additive heritabilities, lactation

532 yield effects may better reflect whole-animal energy and fitness status and be a better proxy of a

533 wider range of underlying biological disorders. At a single locus level, we identified five QTL

534 presenting seven candidate causative variants in the DOCK8, IL4R, KIAA0556, ITGAL, LRCH4,

535 SLC25A4, and RBM34 genes, highlighting medium to large effect recessive variants that may

536 provide future opportunity for diagnostic testing and animal improvement. 


\section{Figure Legends}

\section{Figure 1 - Dominance and additive Manhattan plots for lactation traits.}

539 a-e, Manhattan plots for milk volume (a), milk protein yield (b), milk fat yield (c), milk protein

540 percentage (d), and milk fat percentage (e) showing significance of genotypic dominance (blue

541 and light blue), and additive (grey and light grey) estimates for $\sim 16.6$ million imputed sequence

542 variants. Chromosomes are differentiated by alternating colours and a grey line indicates the

543 false discovery rate of $1 \times 10^{-3}$, used to account for multiple testing. The $y$-axes are truncated for

544 display purposes (indicated by 3 dots).

\section{$545 \quad$ Figure 2}

546 Plots presenting genetic architecture of significant dominance QTL from GWAS on milk volume

547 (milk), milk protein yield (prot), milk fat yield (fat), milk protein percentage (protper), milk fat

548 percentage (fatper), and. The plots contrast the minor allele frequency (MAF) against the

549 dominance effect size (a), and the absolute value of $k$, where $k=d /|a|$ against the dominance

550 effect size (b).

\section{$551 \quad$ Figure 3}

552 Manhattan plots for the five novel milk protein yield QTL representing the chr8:44Mbp (a),

553 chr25:24-27Mbp (b), chr25:35Mbp (c), chr27:15Mbp (d), and chr28:7Mbp (e) loci. Variants are

554 coloured by $\operatorname{LD}\left(R^{2}\right)$ values with the top tag variant per locus, protein coding variants are shown

555 as outlined triangles. Gene tracks are presented below each plot based on Ensembl 97, where

556 gene names have been filtered on size.

\section{$557 \quad$ Figure 4}

558 Plots contrasting minor allele frequency (MAF) and the absolute effect size between homozygote

559 genotype classes (Effect size) for additive (blue) and dominance (red) QTL detected via GWAS

560 across lactation traits. 


\section{Supplementary Figure Legends}

\section{Supplementary Figure 1}

567 Iterative Manhattan plots for milk-protein yield on chromosome 25. Blue indicates the candidate

568 causal variants in genes; IL4R, KIAA0556, and ITGAL, and red indicates the candidate causal

569 variant in the $L R C H 4$ gene. A grey line indicates the false discovery rate of $1 \times 10^{-3}$, used to

570 account for multiple testing.

\section{Supplementary Figure 2}

572 Iterative Manhattan plots for milk-protein yield on chromosome 28. Blue indicates the candidate

573 causal variant in the GALNT2 gene, and red indicates the candidate causal variant in the RBM34

574 gene. A grey line indicates the false discovery rate of $1 \times 10^{-3}$, used to account for multiple testing. 


\section{References}

594 1. Charlier C, Agerholm JS, Coppieters W, Karlskov-Mortensen P, Li W, de Jong G, et al. A

595 Deletion in the Bovine FANCI Gene Compromises Fertility by Causing Fetal Death and

596 Brachyspina. Veitia RA, editor. PLoS One. Public Library of Science; 2012;7:e43085.

597 2. Littlejohn MD, Henty KM, Tiplady K, Johnson T, Harland C, Lopdell T, et al. Functionally

598 reciprocal mutations of the prolactin signalling pathway define hairy and slick cattle. Nat

599 Commun. Nature Publishing Group; 2014;5:1-8.

600 3. Bourneuf E, Otz P, Pausch H, Jagannathan V, Michot P, Grohs C, et al. Rapid Discovery of de

601 Novo Deleterious Mutations in Cattle Enhances the Value of Livestock as Model Species. Sci

602 Rep. Nature Publishing Group; 2017;7:1-19.

603 4. Charlier C, Li W, Harland C, Littlejohn M, Coppieters W, Creagh F, et al. NGS-based reverse

604 genetic screen for common embryonic lethal mutations compromising fertility in livestock.

605 Genome Res. Cold Spring Harbor Laboratory Press; 2016;26:1333-41.

606 5. Michot P, Chahory S, Marete A, Grohs C, Dagios D, Donzel E, et al. A reverse genetic

607 approach identifies an ancestral frameshift mutation in RP1 causing recessive progressive retinal

608 degeneration in European cattle breeds. Genet Sel Evol. BioMed Central Ltd.; 2016;48:1-15.

609 6. Zhu Z, Bakshi A, Vinkhuyzen AAE, Hemani G, Lee SH, Nolte IM, et al. Dominance genetic

610 variation contributes little to the missing heritability for human complex traits. Am J Hum Genet.

611 Cell Press; 2015;96:377-85.

612 7. Bolormaa S, Pryce JE, Zhang Y, Reverter A, Barendse W, Hayes BJ, et al. Non-additive

613 genetic variation in growth, carcass and fertility traits of beef cattle. BioMed Central; 2015;47.

614 8. Sun C, VanRaden PM, Cole JB, O'Connell JR. Improvement of prediction ability for genomic

615 selection of dairy cattle by including dominance effects. Barendse W, editor. PLoS One. Public

616 Library of Science; 2014;9:e103934.

617 9. Aliloo H, Pryce JE, González-Recio O, Cocks BG, Hayes BJ. Accounting for dominance to

618 improve genomic evaluations of dairy cows for fertility and milk production traits. Genet Sel Evol.

619 BioMed Central Ltd.; 2016;48:1-11.

620 10. Jiang J, Ma L, Prakapenka D, VanRaden PM, Cole JB, Da Y. A Large-Scale Genome-Wide 
621 Association Study in U.S. Holstein Cattle. Front Genet. Frontiers Media S.A.; 2019;10:412.

622 11. Reynolds EGM, Neeley C, Lopdell TJ, Keehan M, Dittmer K, Harland CS, et al. Non-additive

623 association analysis using proxy phenotypes identifies novel cattle syndromes. Nat Genet. Nature

624 Publishing Group; 2021;1-6.

625 12. Grisart B, Coppieters W, Farnir F, Karim L, Ford C, Berzi P, et al. Positional candidate cloning

626 of a QTL in dairy cattle: Identification of a missense mutation in the bovine DGAT1 gene with

627 major effect on milk yield and composition. Genome Res. Cold Spring Harbor Laboratory Press;

$628 \quad 2002 ; 12: 222-31$.

629 13. Blott S, Kim JJ, Moisio S, Schmidt-Küntzel A, Cornet A, Berzi P, et al. Molecular dissection of

630 a quantitative trait locus: A phenylalanine-to-tyrosine substitution in the transmembrane domain of

631 the bovine growth hormone receptor is associated with a major effect on milk yield and

632 composition. Genetics. 2003;163:253-66.

633 14. Cohen-Zinder M, Seroussi E, Larkin DM, Loor JJ, Everts-Van Der Wind A, Lee JH, et al.

634 Identification of a missense mutation in the bovine ABCG2 gene with a major effect on the QTL

635 on chromosome 6 affecting milk yield and composition in Holstein cattle. Genome Res. Cold

636 Spring Harbor Laboratory Press; 2005;15:936-44.

637 15. Littlejohn MD, Tiplady K, Lopdell T, Law TA, Scott A, Harland C, et al. Expression variants of

638 the lipogenic AGPAT6 gene affect diverse milk composition phenotypes in Bos taurus. PLoS

639 One. Public Library of Science; 2014;9:85757.

640 16. Littlejohn MD, Tiplady K, Fink TA, Lehnert K, Lopdell T, Johnson T, et al. Sequence-based

641 Association Analysis Reveals an MGST1 eQTL with Pleiotropic Effects on Bovine Milk

642 Composition. Sci Rep. Nature Publishing Group; 2016;6:1-14.

643 17. Bauman DE, Bruce Currie W. Partitioning of Nutrients During Pregnancy and Lactation: A

644 Review of Mechanisms Involving Homeostasis and Homeorhesis. J Dairy Sci. Elsevier;

645 1980;63:1514-29.

646 18. Garrick DJ, Taylor JF, Fernando RL. Deregressing estimated breeding values and weighting

647 information for genomic regression analyses. Genet Sel Evol. BioMed Central; 2009;41:55.

648 19. Li H. Aligning sequence reads, clone sequences and assembly contigs with BWA-MEM. 
649 2013;

650 20. Depristo MA, Banks E, Poplin R, Garimella K V., Maguire JR, Hartl C, et al. A framework for

651 variation discovery and genotyping using next-generation DNA sequencing data. Nat Genet.

$652 \quad 2011 ; 43: 491-501$.

653 21. Browning BL, Zhou Y, Browning SR. A One-Penny Imputed Genome from Next-Generation

654 Reference Panels. Am J Hum Genet. Cell Press; 2018;103:338-48.

655 22. Wang Y, Tiplady K, Johnson TJJ, Harland C, Keehan M, Reynolds E, et al. Evaluating the

656 accuracy of imputed whole-genome sequence data in admixed dairy cattle. Int Conf Quant Genet

657 6. 2020.

658 23. Yang J, Lee SH, Goddard ME, Visscher PM. GCTA: A tool for genome-wide complex trait

659 analysis. Am J Hum Genet. The American Society of Human Genetics; 2011;88:76-82.

660 24. Fernando RL, Garrick D. Genome-Wide Association Studies and Genomic Prediction. 2013.

661 25. Geweke J. Evaluating the Accuracy of Sampling-Based Approaches to the Calculation of

662 Posterior Moments. Bayesian Stat. 1991.

663 26. Bernal Rubio YL, Gualdrón Duarte JL, Bates RO, Ernst CW, Nonneman D, Rohrer GA, et al.

664 Meta-analysis of genome-wide association from genomic prediction models. Anim Genet. John

665 Wiley \& Sons, Ltd (10.1111); 2015;47:36-48.

666 27. Storey JD, Tibshirani R. Statistical significance for genomewide studies. Proc Natl Acad Sci U

667 S A. 2003;100:9440-5.

668 28. Storey JD, Bass AJ, Dabney A, Robinson D. qvalue: Q-value estimation for false discovery

669 rate control. 2020.

670 29. Yang J, Ferreira T, Morris AP, Medland SE, Madden PAF, Heath AC, et al. Conditional and

671 joint multiple-SNP analysis of GWAS summary statistics identifies additional variants influencing

672 complex traits. Nat Genet. Nature Publishing Group; 2012;44:369-75.

673 30. McLaren W, Gil L, Hunt SE, Riat HS, Ritchie GRS, Thormann A, et al. The Ensembl Variant

674 Effect Predictor. Genome Biol. BioMed Central Ltd.; 2016;17:1-14.

675 31. Ng PC, Henikoff S. SIFT: Predicting amino acid changes that affect protein function. Nucleic

676 Acids Res. Oxford Academic; 2003;31:3812-4. 
677 32. Jiang J, Shen B, O'Connell JR, VanRaden PM, Cole JB, Ma L. Dissection of additive,

678 dominance, and imprinting effects for production and reproduction traits in Holstein cattle. BMC

679 Genomics. BioMed Central Ltd.; 2017;18:1-13.

680 33. Lopdell TJ, Tiplady K, Couldrey C, Johnson TJJ, Keehan M, Davis SR, et al. Multiple QTL 681 underlie milk phenotypes at the CSF2RB locus. Genet Sel Evol. BioMed Central; 2019;51:1-16.

682 34. Lopdell TJ, Tiplady K, Struchalin M, Johnson TJJ, Keehan M, Sherlock R, et al. DNA and 683 RNA-sequence based GWAS highlights membrane-transport genes as key modulators of milk 684 lactose content. BMC Genomics. BioMed Central; 2017;18:968.

685 35. Caroli AM, Chessa S, Erhardt GJ. Invited review: Milk protein polymorphisms in cattle: Effect 686 on animal breeding and human nutrition. J Dairy Sci. Elsevier; 2009;92:5335-52.

687 36. Wei WH, Hemani G, Haley CS. Detecting epistasis in human complex traits. Nat. Rev. Genet. 688 Nature Publishing Group; 2014. p. 722-33.

689 37. Visscher PM, Wray NR, Zhang Q, Sklar P, McCarthy MI, Brown MA, et al. 10 Years of GWAS

690 Discovery: Biology, Function, and Translation. Cell Press; Jul 6, 2017 p. 5-22.

691 38. Hiltpold M, Kadri NK, Janett F, Witschi U, Schmitz-Hsu F, Pausch H. Autosomal recessive loci 692 contribute significantly to quantitative variation of male fertility in a dairy cattle population. BMC

693 Genomics 2021 221. BioMed Central; 2021;22:1-19.

694 39. Kearney CJ, Randall KL, Oliaro J. DOCK8 regulates signal transduction events to control 695 immunity. Cell Mol Immunol. Nature Publishing Group; 2017;14:406-11.

696 40. Engelhardt KR, Mcghee S, Winkler S, Sassi A, Woellner C, Lopez-herrera G, et al. Large 697 deletions and point mutations involving DOCK8 in the autosomal recessive form of the hyper-lgE 698 syndrome. J Allergy. 2009;124:1289.

699 41. Randall KL, Lambe T, Johnson AL, Treanor B, Kucharska E, Domaschenz H, et al. Dock8 700 mutations cripple B cell immunological synapses, germinal centers and long-lived antibody 701 production. 2009;

702 42. Lambe T, Crawford G, Johnson AL, Crockford TL, Bouriez-Jones T, Smyth AM, et al. DOCK8 703 is essential for T-cell survival and the maintenance of CD8 + T-cell memory. Eur J Immunol. John 704 Wiley \& Sons, Ltd; 2011;41:3423-35. 
705 43. Zhang Q, Dove CG, Hor JL, Murdock HM, Strauss-Albee DM, Garcia JA, et al. DOCK8

706 regulates lymphocyte shape integrity for skin antiviral immunity. J Exp Med. Rockefeller

707 University Press; 2014;211:2549-66.

708 44. Janssen E, Tsitsikov E, Al-Herz W, Lefranc G, Megarbane A, Dasouki M, et al. Flow

709 cytometry biomarkers distinguish DOCK8 deficiency from severe atopic dermatitis. Clin Immunol.

$710 \quad$ Academic Press Inc.; 2014;150:220-4.

711 45. Shirakawa T, Deichmann KA, Izuhara K, Mao XQ, Adra CN, Hopkin JM. Atopy and asthma:

712 Genetic variants of IL-4 and IL-13 signalling. Immunol. Today. Elsevier Ltd; 2000. p. 60-4.

713 46. Sanders AAWM, de Vrieze E, Alazami AM, Alzahrani F, Malarkey EB, Sorusch N, et al.

714 KIAA0556 is a novel ciliary basal body component mutated in Joubert syndrome. Genome Biol.

715 BioMed Central Ltd.; 2015;16:1-23.

716 47. Zhang J, Teh M, Kim J, Eva M, Cayrol R, Meade R, et al. A loss-of-function mutation in Itgal

717 contributes to the high susceptibility of Collaborative Cross strain CC042 to Salmonella infections.

718 bioRxiv. Cold Spring Harbor Laboratory; 2019;723478.

719 48. Aloor JJ, Azzam KM, Guardiola JJ, Gowdy KM, Madenspacher JH, Gabor KA, et al. Leucine-

720 rich repeats and calponin homology containing 4 (Lrch4) regulates the innate immune response.

721 J Biol Chem. American Society for Biochemistry and Molecular Biology Inc.; 2019;294:1997-

7222008.

723 49. Graham BH, Waymire KG, Cottrell B, Trounce IA, MacGregor GR, Wallace DC. A mouse

724 model for mitochondrial myopathy and cardiomyopathy resulting from a deficiency in the

725 heart/muscle isoform of the adenine nucleotide translocator. Nat Genet. Nature Publishing Group;

$726 \quad 1997 ; 16: 226-34$.

727 50. Kaukonen J, Juselius JK, Tiranti V, Kyttala A, Zeviani M, Comi GP, et al. Role of adenine 728 nucleotide translocator 1 in mtDNA maintenance. Science (80- ). American Association for the

729 Advancement of Science; 2000;289:782-5.

730 51. Palmieri L, Alberio S, Pisano I, Lodi T, Meznaric-Petrusa M, Zidar J, et al. Complete loss-of-

731 function of the heart/muscle-specific adenine nucleotide translocator is associated with

732 mitochondrial myopathy and cardiomyopathy. Hum Mol Genet. Oxford Academic; 2005;14:3079- 
73388.

734 52. Wang X, Ping C, Tan P, Sun C, Liu G, Liu T, et al. hnRNPLL controls pluripotency exit of

735 embryonic stem cells by modulating alternative splicing of Tbx3 and Bptf . EMBO J. EMBO;

$736 \quad 2021 ; 40: e 104729$.

737 53. Raven LA, Cocks BG, Kemper KE, Chamberlain AJ, Vander Jagt CJ, Goddard ME, et al.

738 Targeted imputation of sequence variants and gene expression profiling identifies twelve

739 candidate genes associated with lactation volume, composition and calving interval in dairy cattle.

740 Mamm Genome. Springer US; 2016;27:81-97.

741 54. Karim L, Takeda H, Lin L, Druet T, Arias J a C, Baurain D, et al. Variants modulating the

742 expression of a chromosome domain encompassing PLAG1 influence bovine stature. Nat Genet.

$743 \quad 2011 ; 43: 405-13$.

744 55. Fink T, Tiplady K, Lopdell T, Johnson T, Snell RG, Spelman RJ, et al. Functional confirmation

745 of PLAG1 as the candidate causative gene underlying major pleiotropic effects on body weight

746 and milk characteristics. Sci Rep. Nature Publishing Group; 2017;7:1-8.

747 56. PRJNA656361 Cattle whole genome sequences [Internet]. 2021. Available from:

748 https://www.ncbi.nlm.nih.gov/bioproject/PRJNA656361 


\section{Declarations}

\section{Availability of data and materials}

763 A subset of whole-genome sequences used for imputation of the genotypes presented in this

764 paper have been deposited in the SRA database [56]. Additional data is available on reasonable

765 request with the permission of Livestock Improvement Corporation, contingent on the execution

766 of an appropriate transfer agreement.

\section{Acknowledgements}

768 We are grateful for the use of New Zealand eScience Infrastructure (NeSI) high-performance

769 computing facilities as part of this research. We are additionally grateful to the funders of this

770 research.

\section{Funding}

772 The research was financially supported by the Ministry of Business, Innovation and Employment

773 (MBIE; Wellington, New Zealand) and the Ministry of Primary Industries (MPI; Wellington, New

774 Zealand), who independently co-funded the work through the Endeavour Fund (LICX1802) and

775 (now historical) Primary Growth Partnership research programs, respectively. EGMR is supported

776 by a MPI Postgraduate Scholarship (Wellington, New Zealand) and an AI Rae Centre scholarship

777 (Hamilton, New Zealand). External funders had no role in the analysis or interpretation of the

778 data, or in writing the manuscript.

\section{Author's Contributions}

780 EGMR, DJG, and MDL conceived and designed the experiments. EGMR, TL, KT, YW, CSH,

781 BLH, DJG, and MDL performed or assisted statistical analysis. EGMR, TL, KT, YW, CSH, TJJJ,

$782 \mathrm{CN}, \mathrm{KC}, \mathrm{RGS}, \mathrm{CC}, \mathrm{SRD}, \mathrm{BLH}, \mathrm{RJS}, \mathrm{DJG}$, and MDL contributed materials or analysis tools.

783 EGMR, DJG, and MDL interpreted results. BLH, RJS, DJG, and MDL were involved in

784 supervising the project. EGMR, DJG, and MDL wrote and revised the manuscript. All authors

785 have read and approved the final manuscript.

\section{Ethics Declaration}


787 All animal experiments were conducted in strict accordance with the rules and guidelines outlined

788 in the New Zealand Animal Welfare Act 1999. The majority of genotype and phenotype data were

789 generated as part of routine commercial activities outside the scope of that requiring formal

790 committee assessment (as defined by the above guidelines).

\section{Competing interests}

$792 \mathrm{TL}, \mathrm{YW}, \mathrm{KT}, \mathrm{CSH}, \mathrm{TJJJ}, \mathrm{CN}, \mathrm{KC}, \mathrm{RGS}, \mathrm{CC}, \mathrm{SRD}$, BLH, RJS, MDL are employees of Livestock

793 Improvement Corporation (LIC; Hamilton, New Zealand), a commercial provider of bovine

794 germplasm. Livestock Improvement Corporation is the applicant for several patent applications

795 related to some of the mutations detailed in this article, with EGMR and MDL named inventors

796 on these applications. Specifically, these filed patents relate to genetic testing applications

797 of mutations impacting the DOCK8 (768802), IL4R (768803), KIAA0556 (768804), ITGAL

798 (777216) LRCH4 (768805), and RBM34 (768806) genes. All other authors declare that they have

799 no competing interests.

800

801 

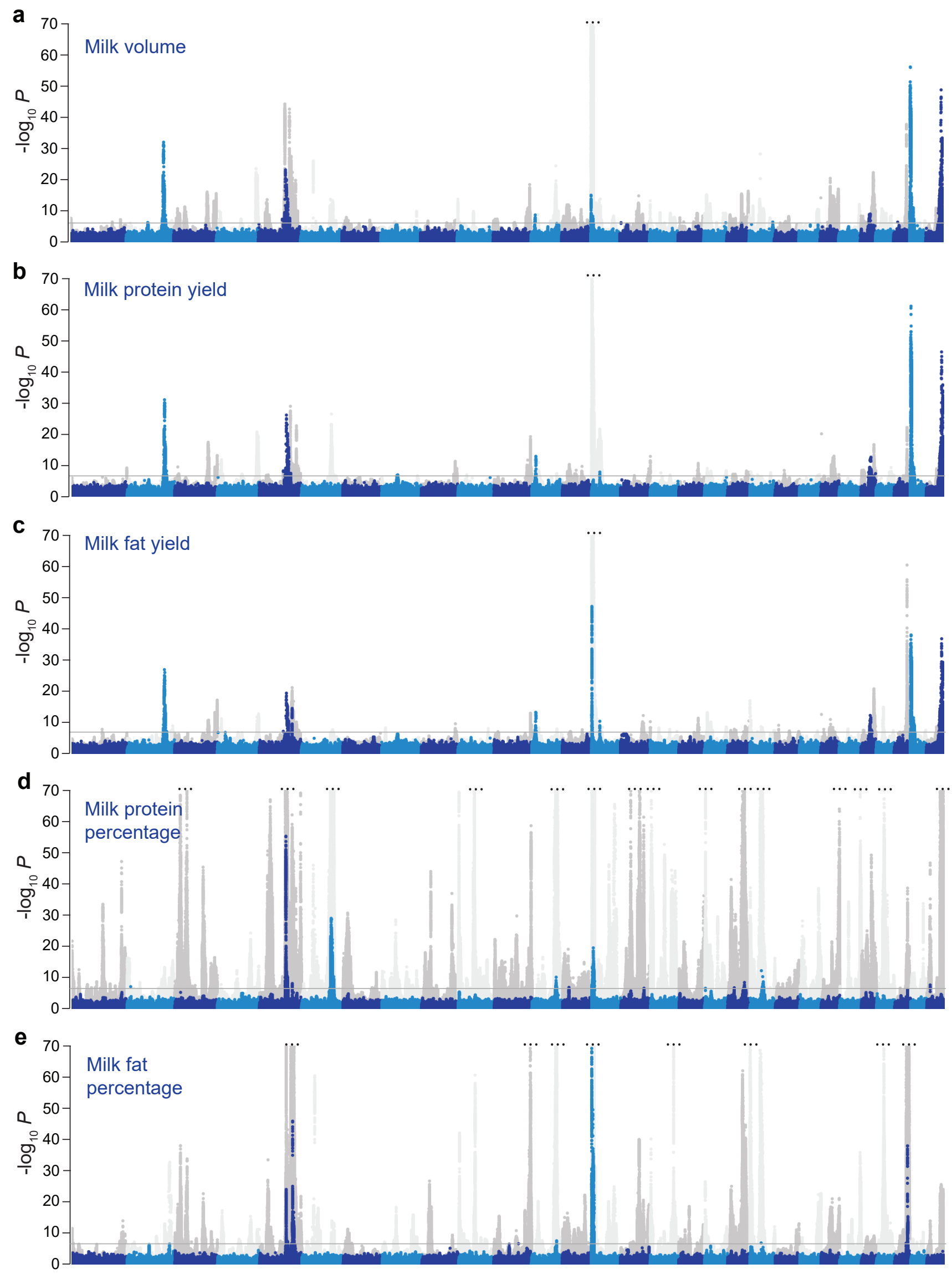
a)

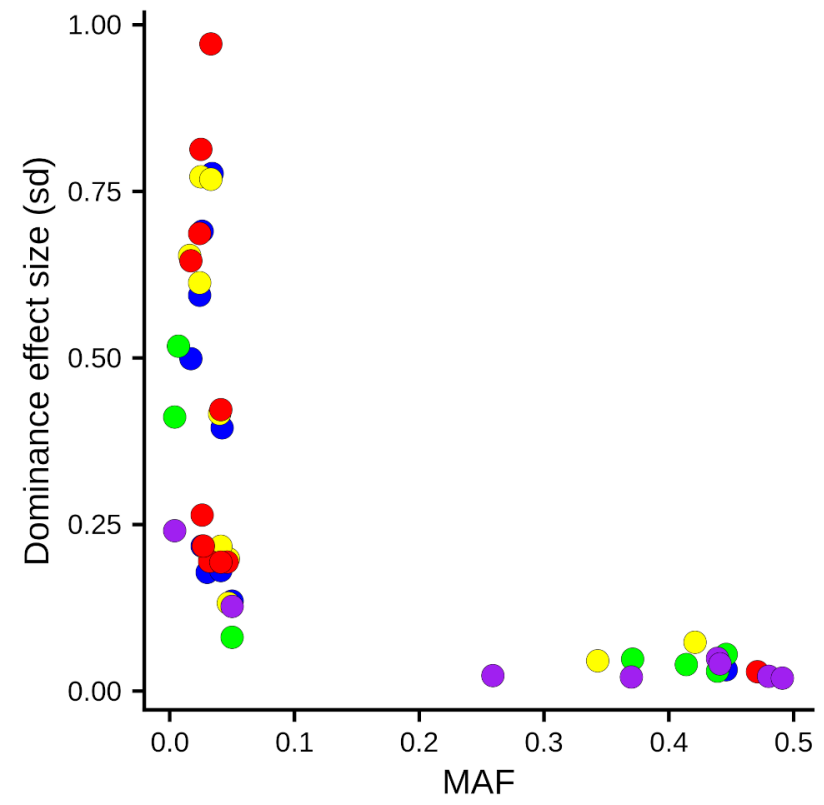

b)

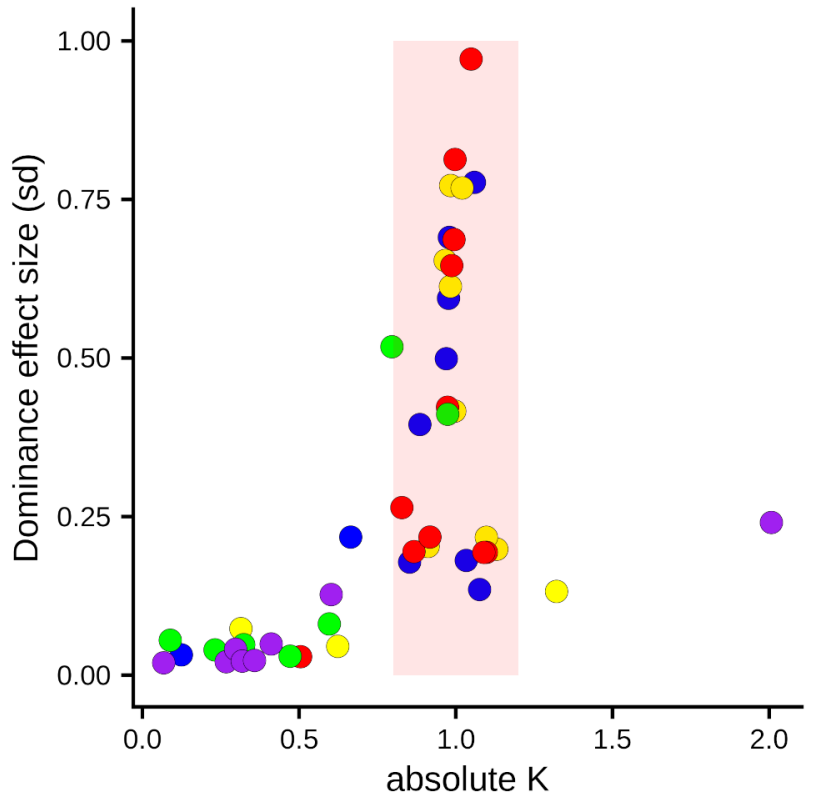


a)

Chromosome 8

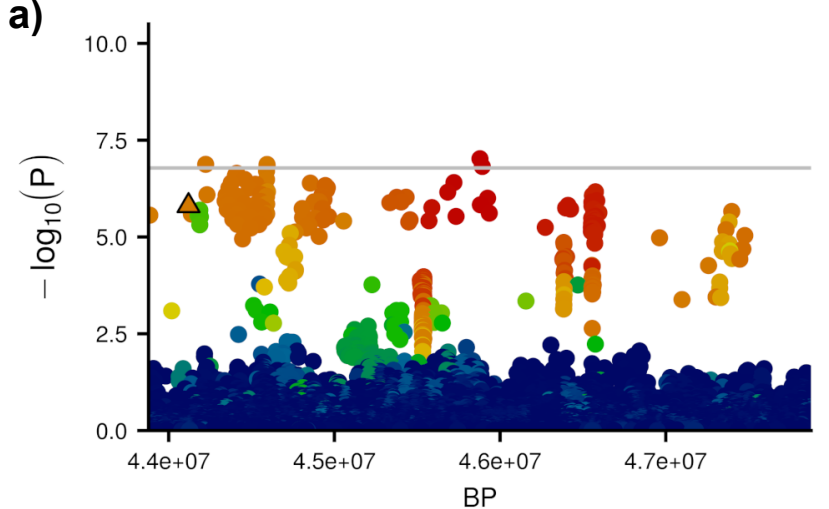

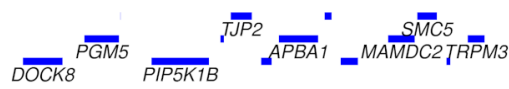

c)

\section{Chromosome 25}

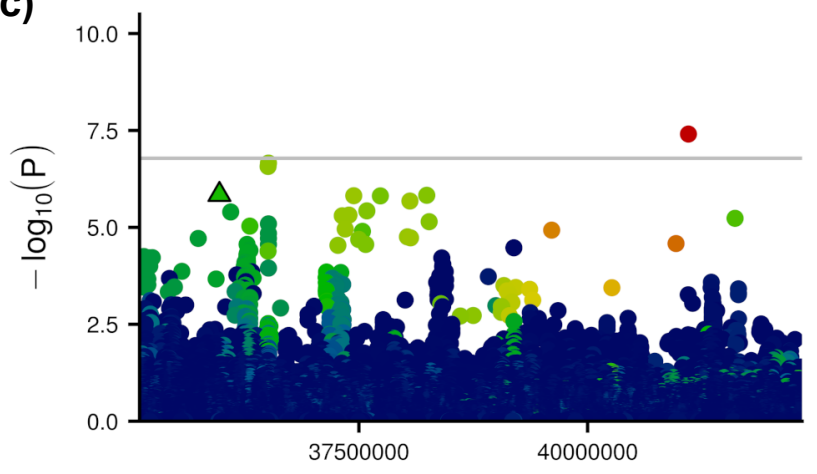

BP

e)

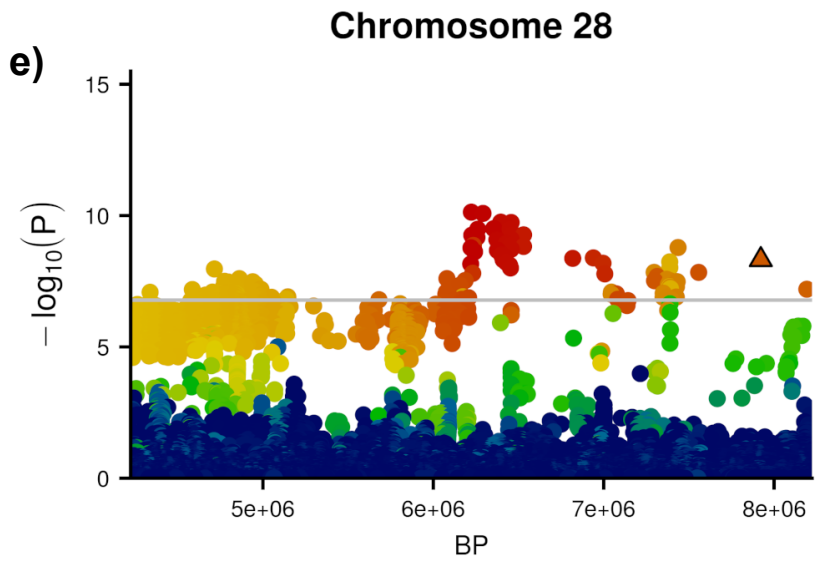

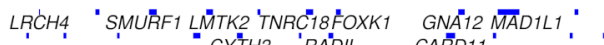

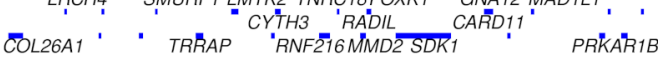

\section{Chromosome 28}

$\mathrm{BP}$ b)

Chromosome 25

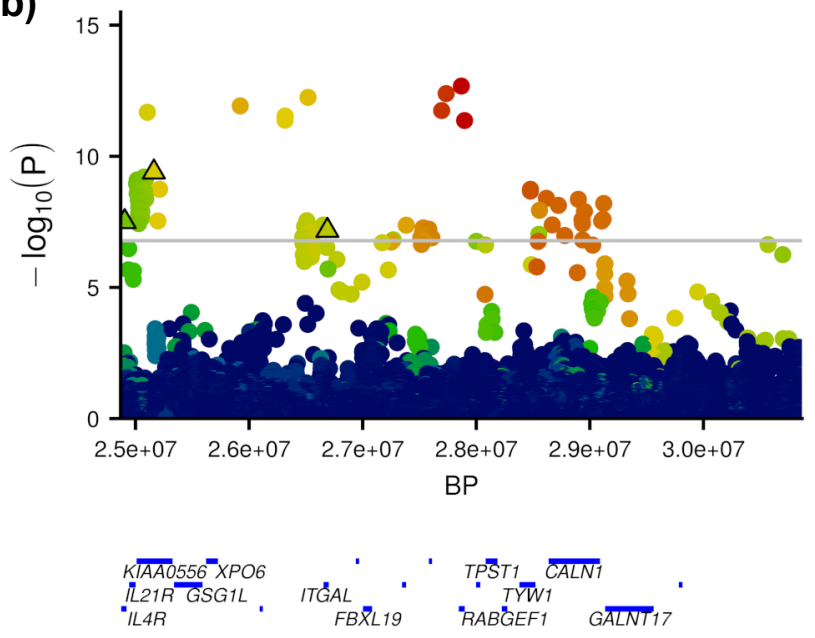

d)

Chromosome 27

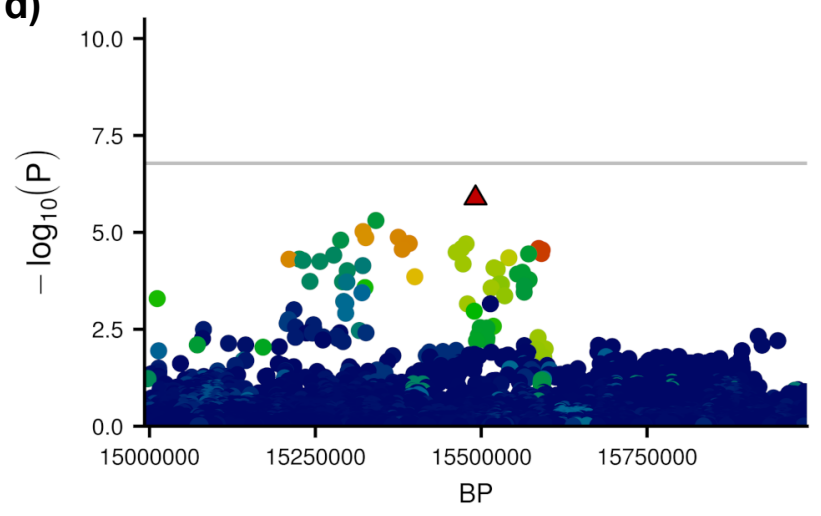

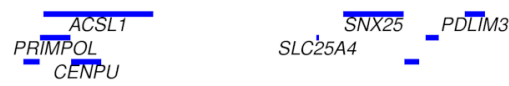

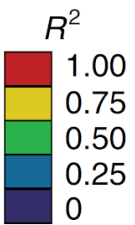

1.00

0.75

0.25 

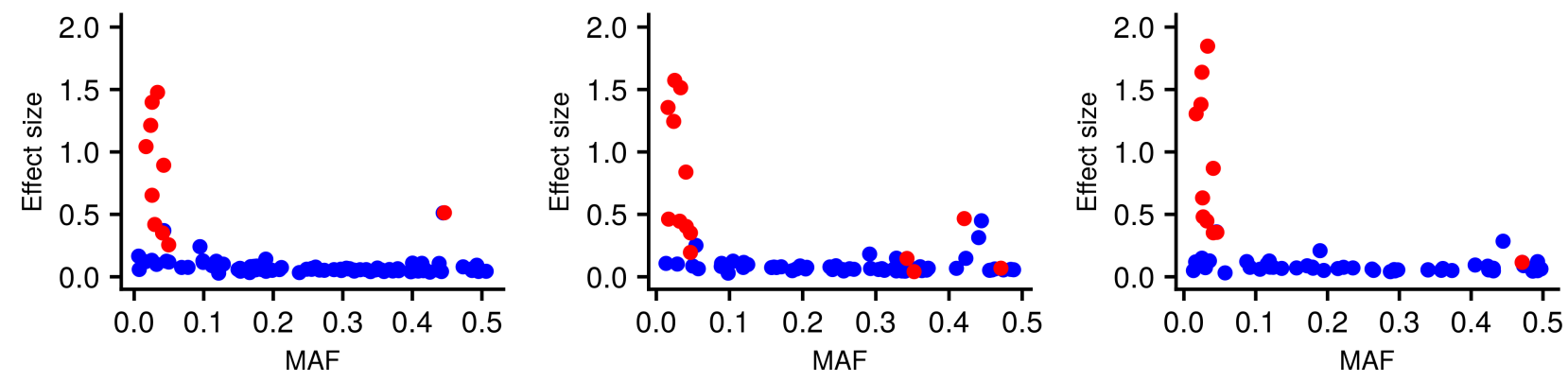

Milk fat percentage

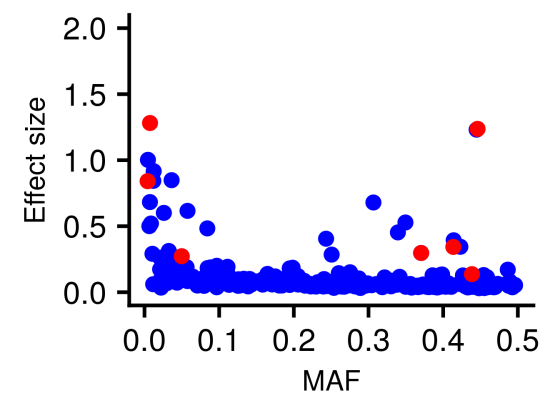

Milk protein percentage

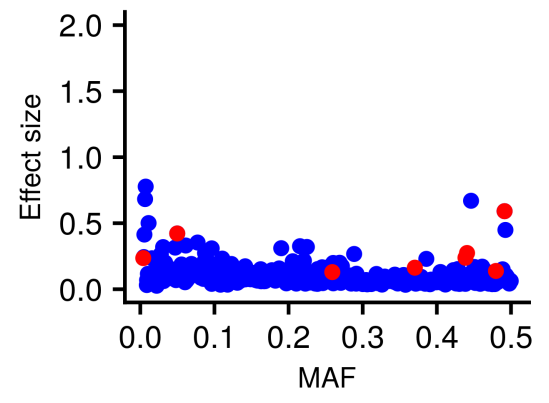


Iteration 0

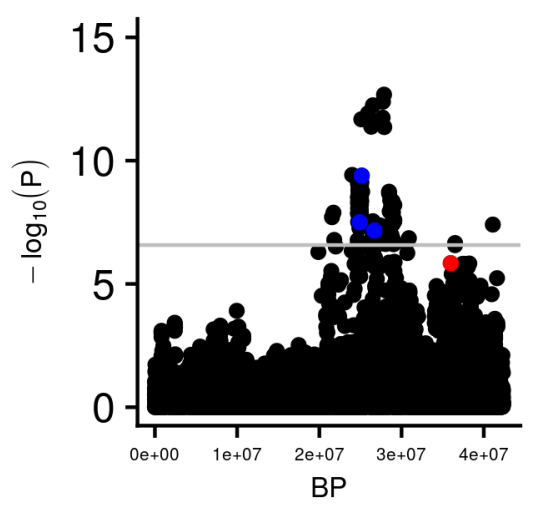

Iteration 1

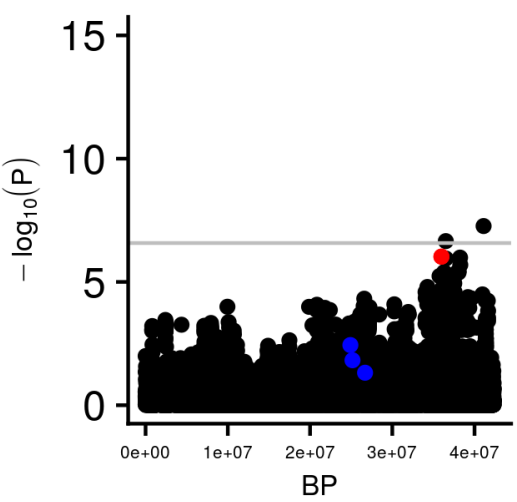

Iteration 2

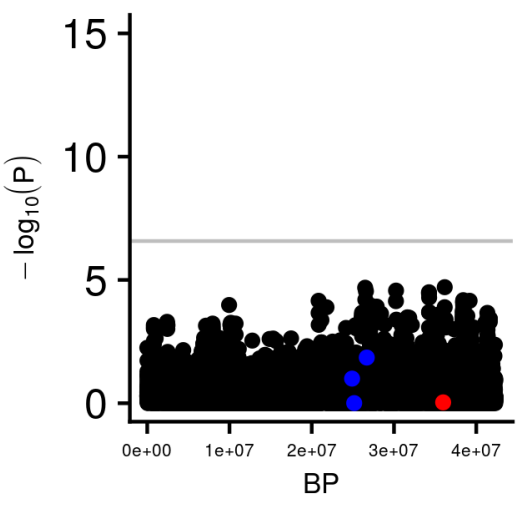


Iteration 0

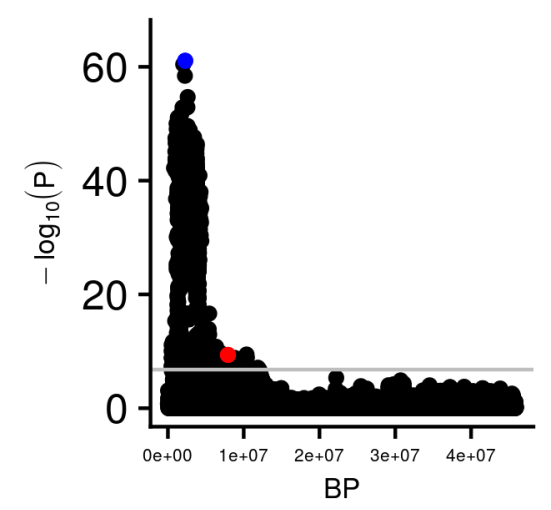

Iteration 1

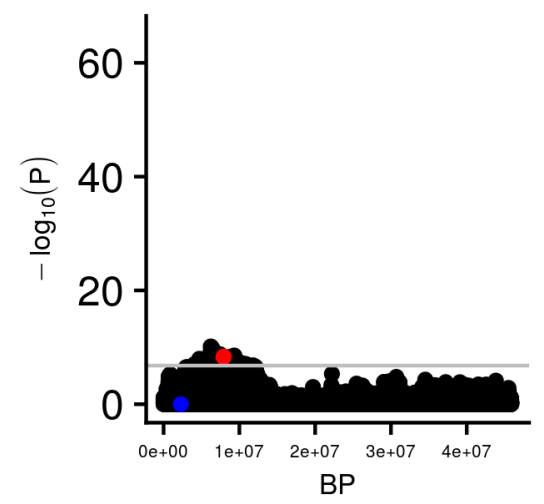

Iteration 2

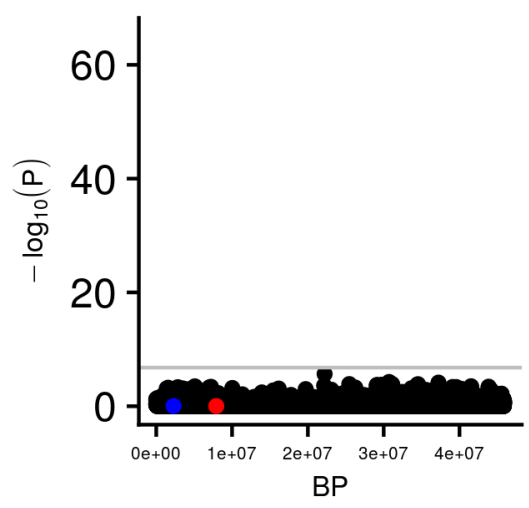

